\title{
Estenose aórtica severa em idosos: avaliação clínica, eletrocardiográfica, ecocardiográfica e angiográfica
}

Severe aortic stenosis in the elderly: clinical, eletrocardiographic, ecocardiographic and angiographic assessment

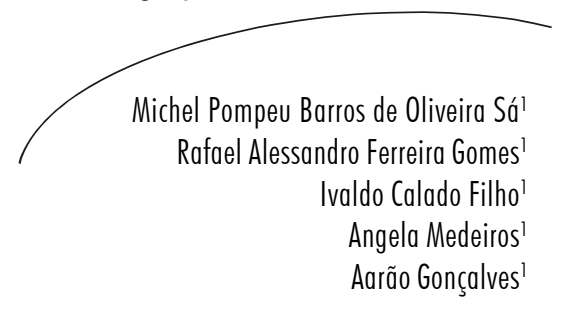

\section{Resumo}

Objetivos: Analisar o perfil clínico e laboratorial de idosos portadores de estenose aórtica severa e possíveis diferenças entre os sexos. Método: Foram estudados 30 pacientes (18 homens e 12 mulheres), com média de idade de $70,7 \pm 5,3$ anos, portadores de estenose aórtica acentuada, sendo avaliados quanto a sintomas clínicos, presença de fatores de risco, padrões eletrocardiográficos, ecocardiográficos e associação com doença arterial coronariana, pela realização de angiografia coronariana. Resultados: Dispnéia foi o sintoma mais frequente (40\%). Mulheres tinham mais angina que os homens. Apenas dois pacientes tinham fração de ejeção baixa. Homens apresentaram menores frações de ejeção que mulheres $(p<0,05)$. Houve alta prevalência $(53,3 \%)$ de doença arterial coronariana associada através de coronariografia. Conclusões: Os achados de dispnéia como sintoma mais frequente no grupo total, diferença da frequência de sintomas entre os sexos e fração de ejeção menor nos homens sugeriram que os mecanismos de adaptação ventricular à estenose aórtica possam ser diferentes entre os sexos. Existe forte associação entre estenose aórtica senil calcificada e doença arterial coronariana em idosos.

\author{
Palavras-chave: \\ Perfil de Saúde. \\ Idoso. Saúde do \\ Idoso. Estenose da \\ Valva Aórtica. \\ Razão de Chances. \\ Gênero e Saúde
}

\footnotetext{
Universidade de Pernambuco, Faculdade de Ciências Médicas. Departamento de Medicina Clínica. Recife, PE, Brasil.
} 
Abstract

Objectives: To evaluate clinical and laboratorial profile in elderly patients with severe aortic stenosis and possible gender differences. Method: We studied 30 patients (18 males and 12 females) aged 70,7 $\pm 5,3$ years with severe aortic stenosis, analyzing clinical manifestations, risk factors, eletrocardiographic and ecocardiographic indexes and association with coronary artery disease by coronary angiographic assessment. Results: Dyspnea was the most frequent symptom with overall prevalence of $40 \%$. Women had more angina than men. Ejection fraction was low in only two patients. Male patients had

Key words: Health Profile. Aged. Health of the Elderly. Aortic Valve Stenosis. Odds Ratio. Gender and Health lower ejection fraction $(\mathrm{p}<0,05)$. High prevalence $(53,3 \%)$ of associated coronary artery disease was observed. Conclusions: Data showed dyspnea as the most common clinical manifestation, gender differences of clinical manifestations and lower ejection fraction in male patients suggest that adaptive mechanisms to this condition may be different in both genders. There is a strong association between aortic stenosis in elderly and coronary artery disease.

INTRODUÇÃO

Para preservar a qualidade de vida com o envelhecimento da população, é importante identificar apropriadamente e tratar as doenças que são encontradas comumente nas pessoas idosas. Estima-se que cerca de 28.000 substituições da válvula aórtica foram realizadas nos EUA em 2004, sendo a estenose aórtica a indicação a mais comum; $61 \%$ destas substituições de válvula aórtica ocorreram em pacientes com mais de 65 anos de idade. ${ }^{1,2}$ Com o desenvolvimento do acesso aos cuidados de saúde, a disponibilidade larga de testes não-invasivos, as técnicas operatórias de baixa mortalidade, o médico da atenção primária e o cardiologista estarão ambos face a face com a necessidade do estabelecimento do diagnóstico e de determinar o tratamento apropriado dos pacientes que têm estenose aórtica.
A doença degenerativa da válvula aórtica (esclerose aórtica) representa muito provavelmente um estágio precoce de um processo patológico que pode resultar na estenose aórtica. O grau de calcificação da válvula aórtica como detectado pela ecocardiografia aumenta com a idade, ocorrendo esclerose aórtica em $20 \%$ da população com idade entre 65 e 74 anos e em $48 \%$ da população com idade acima de 84 anos. ${ }^{3,4}$ Similarmente, a prevalência de estenose aórtica aumenta também com a idade; a estenose aórtica está atualmente presente em 1,3\% da população idosa com 65-74 anos e em $4 \%$ da população com mais de 85 anos de idade. Os médicos devem, consequentemente, ter um ponto inicial relativamente baixo para obter um ecocardiograma em um paciente idoso com um sopro sistólico.

Além da idade, diversos outros fatores de risco foram associados com a esclerose 
aórtica, incluindo o sexo masculino, a lipoproteína (a), a altura, a fração da lipoproteína de baixa densidade de colesterol (LDLc) e diabetes mellitus. ${ }^{3,5-8}$ O efeito que a atuação na modificação destes fatores de risco tem no desenvolvimento da esclerose aórtica e a progressão subsequente da doença permanece obscuro.

A estenose aórtica valvar é uma doença gradualmente progressiva, de modo que os pacientes podem permanecer assintomáticos por anos. É comum o diagnóstico ser considerado inicialmente durante uma consulta de rotina, quando um sopro sistólico é detectado. $\mathrm{O}$ início dos sintomas marca o curso da doença e é de suma importância identificar isto, uma vez que o prognóstico de pacientes sintomáticos é pobre, sem tratamento cirúrgico.

Os sintomas cardinais da estenose aórtica são:

- angina pectoris;

- síncope ou pré-síncope;

- dispnéia

Aproximadamente $50 \%$ dos pacientes com estenose aórtica têm doença arterial coronariana associada para explicar o desenvolvimento de angina. ${ }^{9}$ Inversamente, a ausência de dor torácica não exclui a presença de doença arterial coronariana significativa. Nos pacientes que experimentam angina na ausência de doença coronária, um desequilíbrio entre suprimento e demanda miocárdica de oxigênio é a explanação proposta. A demanda aumentada ocorre secundária à pressão na cavidade ventricular esquerda elevada, prolongado tempo ventricular esquerdo de ejeção e massa aumentada do músculo; vários mecanismos que danificam o fluxo coronário do sangue e limitam o suprimento de oxigênio foram descritos. ${ }^{10,11}$

Síncope ou pré-síncope geralmente são relacionados temporalmente ao exercício. Ocorre quando a pressão ventricular esquerda elevada da cavidade estimula baro-receptores, que induzem à hipotensão arterial, diminuindo o retorno venoso e, ocasionalmente, bradicardia. ${ }^{12}$ Síncope em repouso é em geral resultado de arritmias ventriculares.

Dispnéia pode ser um sintoma de vários processos da doença, incluindo doença arterial coronariana, doença pulmonar obstrutiva crônica, hipertensão e estenose aórtica. Embora pacientes assintomáticos manifestem frequentemente dispnéia como sintoma inicial, geralmente considera-se ocorrer ulteriormente no processo da doença e indica estenose aórtica severa. Os pacientes idosos podem gradualmente reduzir seu nível de atividade em um esforço para evitar a dispnéia e, assim, estarem inconscientes de sua limitação atual, a menos que questionado especificamente.

A incidência de doença coronariana em pacientes submetidos à troca de valva aórtica é estimada entre 7\% e 66\%. Em uma série brasileira recentemente publicada, com pacientes que tinham média de idade de aproximadamente 70 anos, mostrou-se prevalência de $40 \%$ de doença coronariana em 
pacientes com estenose aórtica. Verifica-se certa correlação entre idade e taxa de coronariopatia, em que a faixa etária maior tem maior probabilidade de doença coronariana associada a estenose aórtica. ${ }^{13-17}$

A presença de doença coronariana em pacientes com estenose aórtica tem sido motivo de muitos estudos, e alguns autores têm sugerido a correlação entre fatores de risco (hipertensão, diabetes, dislipidemias, tabagismo) e presença de coronariopatia no paciente valvar aórtico. ${ }^{14,18-20}$

\section{MÉTODOS}

Foram estudados 30 pacientes, com média de idade de 70,7 $\pm 5,3$ anos, que foram internados no Pronto-Socorro Cardiológico de Pernambuco, entre fevereiro e novembro de 2007. Dezoito deles eram do sexo masculino ( $73,2 \pm 5,0$ anos) e 12 do feminino $(71,3 \pm 5,0$ anos), constituindo os dois conjuntos analisados de forma comparativa. Foram apenas incluídos portadores de estenose aórtica acentuada (área valvar ao ecodopplercardiograma $<1,0 \mathrm{~cm}^{2}$ e/ou gradiente transvalvar $>50 \mathrm{mmHg}$ ), que não apresentavam outras afecções valvares, insuficiência renal e doenças consumptivas.

A frequência cardíaca e o ritmo do coração foram avaliados em ambos os sexos. Quanto às características clínicas, procurouse caracterizar a presença das três manifestações mais comuns da estenose aórtica: dispnéia de esforço, angina e síncope.
Foi também analisada a presença de fatores de risco para aterosclerose prévios ao período intra-hospitalar (hipertensão arterial sistêmica - HAS, diabetes mellitus - DM, dislipidemias - DLP, tabagismo), uma vez que estes também são reconhecidos como possíveis fatores de risco para o desenvolvimento de estenose aórtica senil calcificada.

Para avaliação eletrocardiográfica, utilizou-se eletrocardiógrafo de 12 derivações. Para análise de sobrecarga ventricular esquerda (SVE), foram realizados cálculo do índice de Sokolow-Lyon $(>35 \mathrm{~mm})$ e índice de Cornell ( $>28 \mathrm{~mm}$ para homens e $>20 \mathrm{~mm}$ para mulheres). ${ }^{21}$

$\mathrm{Na}$ avaliação ecodopplercardiográfica, foram avaliadas as seguintes variáveis: fração de ejeção (FE), índice de massa (M) expresso em $\mathrm{g} / \mathrm{m}^{2}$ (calculado a partir da relação entre espessura do septo interventricular, da parede posterior e o diâmetro diastólico do $\mathrm{VE}),{ }^{22}$ o gradiente transvalvar aórtico máximo $(G)$ e a área valvar aórtica. ${ }^{23}$

Foi também avaliada a presença de doença arterial coronária importante concomitante à estenose aórtica através de cateterismo cardíaco. Foram considerados coronarianos quando apresentavam pelo menos uma artéria subepicárdica com processo aterosclerótico com mais de $50 \%$ de redução do lúmen vascular.

Todos os dados foram coletados a partir dos prontuários. Este trabalho foi aprovado pelo comitê de ética da instituição. 
$\mathrm{Na}$ análise estatística dos resultados finais obtidos nos dois grupos, sexualmente diversos, estes foram comparados utilizando-se teste de Wilcoxon. $\mathrm{Na}$ análise da associação dos fatores de risco com doença coronariana, foi utilizado teste $t$ de Student. Os dados foram plotados e analisados em software EPI.INFO versão 6.0.1. As diferenças foram consideradas como estatisticamente significantes quando $\mathrm{p}<0,05$.

\section{RESULTADOS}

A incidência das três grandes manifestações clínicas encontra-se na figura 1.
O sintoma mais frequente foi dispnéia aos médios ou grandes esforços, presente em 9 de 18 (50\%) homens e em 3 de 12 (25\%) mulheres, ou seja, em 12 (40\%) do total de pacientes.

Angina de peito foi observada em um $(5,55 \%)$ dos homens e em quatro $(33,3 \%)$ das mulheres. A associação de dispnéia e de angina foi a segunda manifestação clínica em frequência, comparecendo em 10 (33,3\%) dos pacientes, com incidência igual em ambos os sexos. Síncope se apresentou apenas na forma isolada em dois pacientes $(10 \%$ do total), sendo um do sexo masculino e um do sexo feminino.

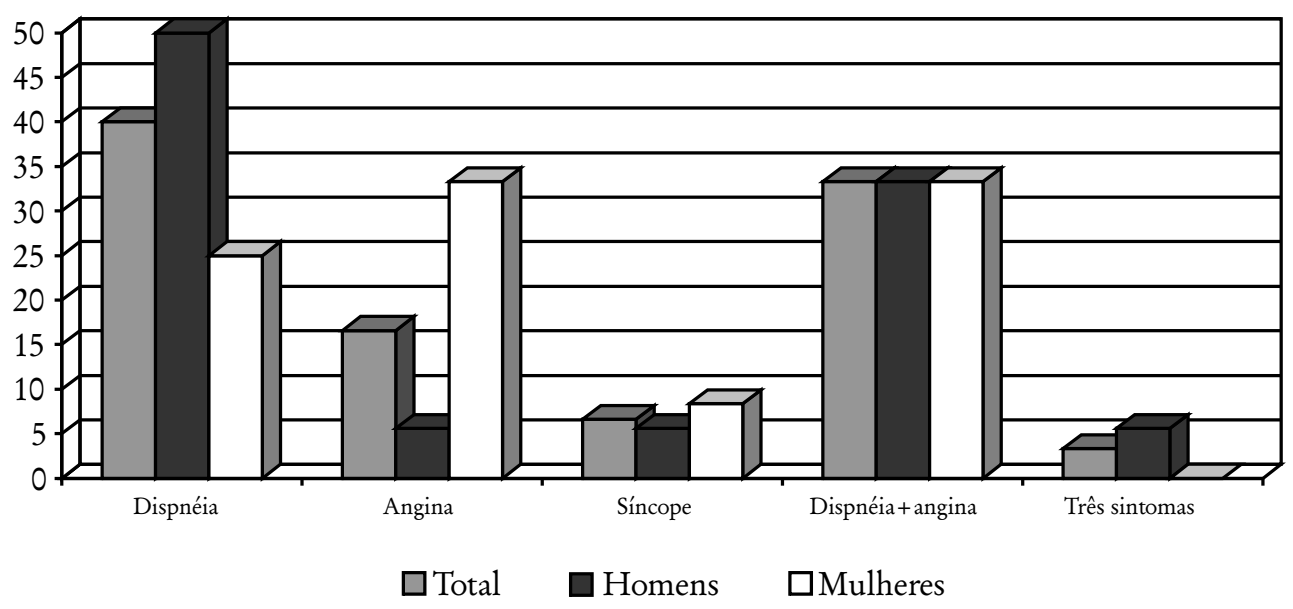

Figura 1- Frequência (\%) dos sintomas clínicos (isolados e associados). 2008. 
A análise dos fatores de risco para aterosclerose prévios ao internamento estão relatadas na figura 2. Observamos prevalência de
70\% para hipertensão arterial, 33,3\% para diabetes, $60 \%$ para dislipidemia e $50 \%$ para tabagismo.

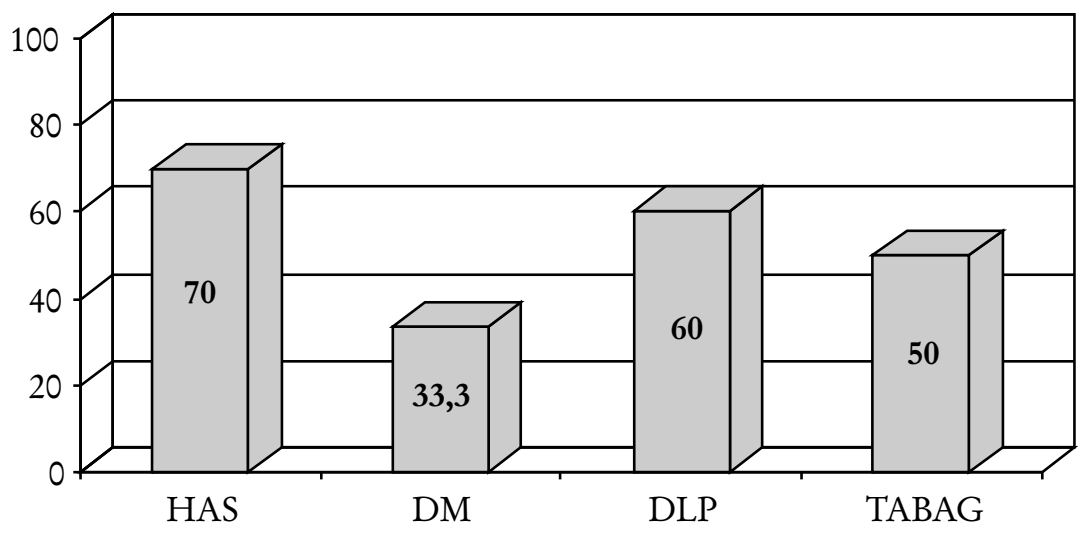

Figura 2 - Frequência (\%) dos fatores de risco para aterosclerose. 2008.

O exame eletrocardiográfico evidenciou sobrecarga atrial esquerda em 14 (46,6\%) pacientes, sobrecarga ventricular esquerda em $16(53,3 \%)$ pacientes pelo critério de Sokolow-Lyon e em 23 (76,6\%) pelo critério de Cornell, bloqueio de ramo esquerdo em dois $(6,6 \%)$, bloqueio de ramo direito em quatro (13,3\%), bloqueio divisional ântero-superior esquerdo em seis (20\%). Todos os pacientes apresentavam alterações do segmento ST-T e ondas T negativas. Dois dos pacientes ( 1 homem e 1 mulher) apresentavam fibrilação atrial e os outros ritmo sinusal regular. ${ }^{21}$

Dos 16 pacientes que apresentavam índice de Sokolow positivo, ${ }^{21}$ dois não apresentavam HVE ao ecocardiograma (12,5\% de falsos positivos). Dentre os 14 pacientes nos quais o índice de Sokolow foi negativo, oito apresentavam HVE ao ecocardiograma ( $57,1 \%$ de falso negativos). Dos 23 pacientes que apresentavam índice de Cornell positivo, um não apresentava HVE ao ecocardiograma (4,3\% de falsos positivos). Dos sete pacientes com Cornell negativo, seis pacientes confirmaram a ausência de HVE ao ecocardiograma ( $14,5 \%$ de falsos negativos).

O estudo pela ecodopplercardiografia revelou que apenas dois $(6,6 \%)$ pacientes apresentavam $\mathrm{FE}<0,50$. Átrio esquerdo $>4 \mathrm{~cm}$ esteve presente em $19(63,3 \%)$ pacientes e calibre da aorta $>3,5 \mathrm{~cm}$ em 12 $(40 \%)$. Índice de massa $>150 \mathrm{~g} / \mathrm{m}^{2}$ foi observado em 21 (70\%) pacientes. A área valvar média do grupo total de pacientes foi de $0,7 \mathrm{~cm}^{2}$. A análise das variáveis ecocardio- 
gráficas (tabela 1) evidenciou diferenças estatisticamente significativas entre os sexos para os valores da fração de ejeção.

A relação entre os sintomas clínicos e os achados ecodopplercardiográficos encontram-se nas figuras 3 e 4 . Evidenciou-se que os pacientes com dispnéia apresentavam massa ventricular significantemente maior $(p<0,05)$ e FE expressivamente menor $(p<0,05)$ que os demais. Por outra, os portadores de angina de peito exibiram massa ventricular esquerda significantemente menor $(p<0,05)$ e maior FE que o restante.

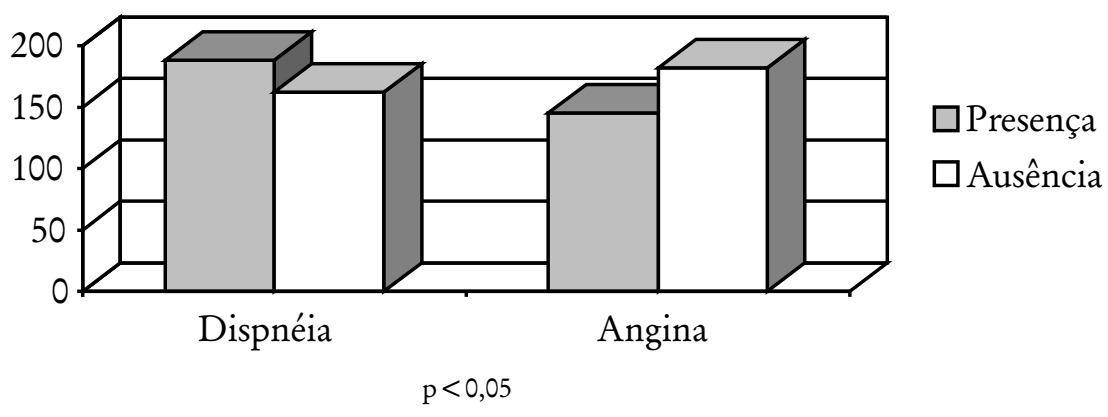

Figura 3 - Relação do índice de massa ventricular $(\mathrm{g} / \mathrm{m} 2)$ ao ecodopplercardiograma com os sintomas dispnéia e angina.

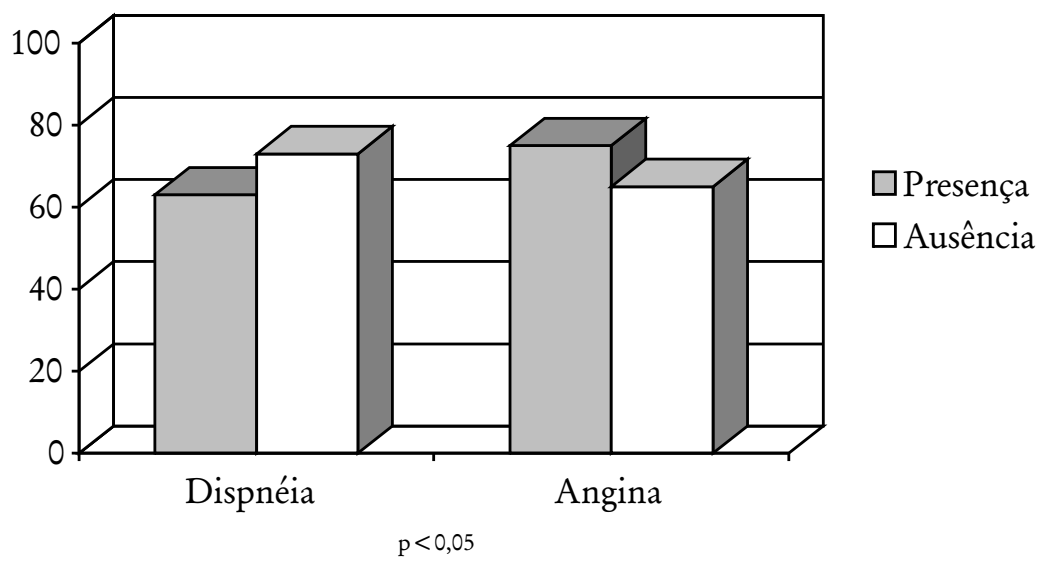

Figura 4 - Relação da fração de ejeção (\%) ao ecodopplercardiograma com os sintomas dispnéia e angina. 2008. 
Quando da análise da presença de doença arterial coronária importante concomitanteà estenose aórtica severa através de cateterismo, dos 30 pacientes do estudo, $16(53,3 \%)$ apresentavam lesões importantes nas artérias coronárias que necessitavam de revascularização miocárdica. Deste grupo, sete $(43,75 \%)$ pacientes não apresentavam angina. Não houve associação significativa entre os grupos com e sem doença coronariana em relação ao sexo, número de fatores de risco, HAS, DM, dislipidemia e tabagismo.

\section{DISCUSSÃO}

Nas manifestações clínicas analisadas, verificou-se que o sintoma mais frequente foi a dispnéia, seguido por associação de dispnéia e angina e, finalmente, por angina de peito isolada. Esses achados estão em desacordo com os observados na maior parte dos trabalhos publicados em que a angina de peito surge como o sintoma mais frequente, independentemente de qualquer associação com doença coronária, mas concordam com o observado por outros que também encontraram dispnéia como o sintoma mais frequente. ${ }^{24-28}$ Deve ser salientado que, contrariamente à literatura, síncope ocorreu em pequena proporção.

A análise dos sintomas de acordo com o sexo demonstrou que os homens apresentaram maior frequência de dispnéia, seguida da associação de angina e dispnéia, e as mulheres apresentaram angina isolada (em maior frequência que homens) e a associa- ção angina mais dispnéia na mesma proporção que o grupo do sexo masculino.

A relação entre valores maiores de índice de massa $(\mathrm{p}<0,05)$ e menores da FE $(\mathrm{p}<0,05)$ com o achado de dispnéia sugere desadaptação ventricular esquerda perante a sobrecarga de pressão. De fato, a hipertrofia desta câmara é o mecanismo que diminui a tensão e o estresse da parede ventricular. ${ }^{28,29}$ Além disso, esse achado pode ser justificado por alterações de relaxamento do músculo cardíaco hipertrofiado, causas primárias dos sintomas de congestão pulmonar; ocorrendo mais tarde, no estágio final do processo, ocorre má adaptação ventricular esquerda com dilatação.

Outro fator de queda da função sistólica nesse grupo de pacientes seria a redução intrínseca da contratilidade miocárdica observada em sobrecargas de pressão. ${ }^{30}$ Finalmente, a presença de fibrose miocárdica, secundária à própria hipertrofia ventricular, contribuiria para a alteração hemodinâmica.

Na nossa casuística, entretanto, foram encontrados apenas dois $(6,6 \%)$ pacientes com $\mathrm{FE}<0,50$. Sabemos, no entanto, que valores normais desse fator em estados de insuficiência cardíaca foram descritos em idosos, ${ }^{31}$ sendo então valorizada a disfunção diastólica do VE na redução da capacidade funcional.

Nossos dados revelaram, ainda, que pacientes com angina de peito apresentaram valores de índice de massa expressivamente menores $(p<0,05)$ e valores de FE significativamente maiores $(p<0,05)$, em comparação com o grupo não-anginoso (figura 4). Este 
achado sugere que os pacientes com angina apresentaram mecanismos adaptativos funcionais miocitários exacerbados (como por exemplo, o aumento do consumo de oxigênio para maior desenvolvimento de trabalho miocárdico para manutenção da fração de ejeção), frente à sobrecarga de pressão, em detrimento do aumento de substrato anatômico.

Com a eletrocardiografia convencional, foi possível fazer diagnóstico de HVE (índice de Sokolow $>35 \mathrm{~mm})$ em $16(53,3 \%)$ de $30 \mathrm{pa}-$ cientes. Destes, dois não apresentavam índice de massa $>150 \mathrm{~g} / \mathrm{m} 2$ (12,5\% de falsos positivos). Dentre os 14 pacientes nos quais o índice foi $<35 \mathrm{~mm}$, oito apresentavam valor de massa $>150 \mathrm{~g} / \mathrm{m} 2$ ( $57,1 \%$ de falso negativos). Esse resultado sugere que a presença desse sinal, ao ECG convencional, não é constante no diagnóstico de EAo em idosos. É possível que a falta de diagnóstico de sobrecarga ventricular esquerda, por meio da eletrocardiografia, esteja relacionada à baixa sensibilidade do índice de Sokolow, quando comparado com outros métodos eletrocardiográfi$\cos ,{ }^{15,21}$ como por exemplo o índice de Cornell. Dos 23 pacientes que apresentavam índice de Cornell positivo, um não apresentava HVE ao ecocardiograma ( $4,3 \%$ de falsos positivos). Dos sete pacientes com Cornell negativo, seis confirmaram a ausência de HVE ao ecocardiograma (14,5\% de falsos negativos). Essa diferença mostrou que o índice de Cornell foi muito mais sensível e específico para HVE do que o índice de Sokolow. ${ }^{21}$

A presença de bloqueios de ramo, direito ou esquerdo e divisionais do ramo esquerdo ocorreram em menor proporção que o relatado pela literatura. $\mathrm{O}$ mesmo foi observado com a fibrilação atrial. ${ }^{32-34}$

A análise de associação com possíveis fatores de risco, evidentemente, só pôde ser efetivada com alguma cautela, partindo-se do ponto de que não foi realizado estudo comparativo com grupo controle (sem lesão aórtica), o que limita o alcance de certas considerações. Os dados obtidos, entretanto, justificam uma pequena citação.

Em 60\% dos pacientes, havia história pregressa de dislipidemia; em $50 \%$, havia história de tabagismo e em $70 \%$, hipertensão. Tais poderiam ser analisados à luz da conhecida hipótese de que o processo de calcificação valvar senil estaria relacionado a perturbações lipídicas, estresse de cisalhamento aumentado e agressões externas, sendo então representativo de estado comparável ao da aterosclerose. ${ }^{35,36}$

No estudo dos resultados ecodopplercardiográficos segundo os sexos, pôde ser evidenciado que, apesar de o índice de massa e os gradientes de pressão não diferirem entre eles, as mulheres apresentaram FE maiores que os observados nos homens $(\mathrm{p}<0,05)$ (tabela 1).

Estes achados devem ser comparados aos obtidos por outro estudo ${ }^{37}$ sobre portadores de afecção valvar e com idades $>60$ anos. As idosas apresentaram função sistólica bem preservada e os idosos tinham tendência a maior HVE, com valores reduzidos de capacidade 
Tabela 1 - Parâmetros ecocardiográficos distribuídos por sexo.

\begin{tabular}{cccc}
\hline & $\mathrm{M}\left(\mathrm{g} / \mathrm{m}^{2}\right)$ & $\mathrm{FE}(\%)$ & $\mathrm{G}(\mathrm{mmHg})$ \\
\hline Mulheres & $173 \pm 40$ & $76 \pm 7 *$ & $69 \pm 15$ \\
Homens & $175 \pm 39$ & $66 \pm 9 *$ & $66 \pm 12$ \\
\hline $\mathrm{p}<0,05$ & &
\end{tabular}

sistólica. Todos esses dados sugerem, acentuadamente, uma real diferença sexual no mecanismo de adaptação crônica do VE à sobrecarga lenta de pressão, com maior capacidade das mulheres para manter a função ventricular em níveis mais elevados.

Em nossa casuística, a prevalência de $53,3 \%$ de doença coronariana mostrou-se dentro da faixa referida pela literatura. Uma metanálise com 33 estudos mostrou $37 \%$ de prevalência de doença coronariana em pacientes com estenose aórtica calcificada, ${ }^{14} \mathrm{com}$ uma variância de $7 \%$ a $66 \%$. Alguns trabalhos acharam incidência de fatores de risco alta em pacientes com coronariopatia associada à estenose aórtica, enquanto a ausência de fatores de risco e angina foram suficientes para excluir doença coronariana com a valvopatia em outro estudo. ${ }^{13,20,38} \mathrm{Na}$ nossa análise, o maior número de fatores de risco não aumentou a incidência de coronariopatia e a ausência de angina não excluiu a presença de doença coronariana (uma vez que $43,75 \%$ dos que tinham lesões coronarianas importantes não tinham angina).
Este estudo apresentou como limitações o número amostral de pacientes pequeno e ausência de grupo controle (sem lesão aórtica) para que pudéssemos demonstrar de forma direta e objetiva a influência dos fatores de risco para aterosclerose no desenvolvimento da estenose aórtica senil calcificada.

Os resultados permitiram concluir que o perfil dos pacientes idosos com EAo severa caracterizou-se por apresentar: a) dispnéia como sintoma mais frequente em homens e angina mais frequente em mulheres; $b$ ) menor índice de massa e maiores $\mathrm{FE}$ em pacientes com angina de peito; c) $\mathrm{FE}>0,50$, na grande maioria dos pacientes, o que ressalta o valor da disfunção ventricular diastólica do idoso no desenvolvimento dos sintomas de congestão pulmonar; d) as mulheres com capacidade para manter maiores frações de ejeção que homens; e) o índice de Cornell mais sensível e mais específico que o índice de Sokolow na identificação da HVE; f) alta prevalência de doença coronariana concomitante a EAog) a ausência de angina como não preditora de ausência de doença coronariana importante. ${ }^{21}$ 


\section{REFERÊNCIAS}

1. Vital and health statistics, series 13 . National Center for Health Statistics 2005;127.

2. Selzer A. Changing aspects of the natural history of valvular aortic stenosis. N Engl J Med 1987;317:91-8.

3. Stewart BF, Siscovick D, Lind BK, et al. Clinical factors associated with calcific aortic valve disease. J Am Coll Cardiol 1997;29:630-4.

4. Lindroos M, Kupari M, Heikkila J, Tilvis R. Prevalence of aortic valve abnormalities in the elderly: an echocardiographic study of a random population sample. J Am Coll Cardiol 1993;21:1220-5.

5. Deutscher S, Rockette HE, Krishnaswami V. Diabetes and hypercholesterolemia among patients with calcific aortic stenosis. J Chronic Dis 1984;37:407-15.

6. Aronow WS, Kronzon I. Correlation of prevalence and severity of valvular aortic stenosis determined by continuous-wave Doppler echocardiography with physical signs of aortic stenosis in patients aged 62 to 100 years with aortic systolic ejection murmurs. Am J Cardiol 1987;60:399-401.

7. Mohler ER, Sheridan MJ, Nichols R, Harvey WP, Waller BF. Development and progression of aortic valve stenosis: atherosclerosis risk factors-a causal relationship? A clinical morphologic study. Clin Cardiol 1991;14:995-9.

8. Gotoh T, Kuroda T, Yamasawa M, et al. Correlation between lipoprotein(a) and aortic valve sclerosis assessed by echocardiography (the JMS cardiac echo and cohort study). Am J Cardiol 1995;76:928-32.

9. Julius BK, Spillmann M, Vassalli G, Villari B, Eberli FR, Hess OM. Angina pectoris in patients with aortic stenosis and normal coronary arteries. Mechanisms and pathophysiological concepts. Circulation 1997;95:892-8.

10. Marcus ML, Doty DB, Hiratzka LF, Wright CB, Eastham CL. Decreased coronary reserve: a mechanism for angina pectoris in patients with aortic stenosis and normal coronary arteries. N Engl J Med 1982;307:1362-6.

11. Lombard JT, Selzer A. Valvular aortic stenosis. A clinical and hemodynamic profile of patients. Ann Intern Med 1987;106:292-8.

12. Richards AM, Nicholls MG, Ikram H, Hamilton EJ, Richards RD. Syncope in aortic valvular stenosis. The Lancet. 1984;2:1113-6.

13. Pluta W, Buszman P, Lekston A, Pasyk S. Coronary artery stenosis in pacients with vascular heart disease. Cor Vasa 1989;31:451-7.

14. Mauter GC, Roberts WC. Reported frequency of coronary arterial narrowing by angiogram in patient with valvular aortic stenosis. Am J Cardiol 1992;69:539-40.

15. Rangel MC, Grinberg M, Maranhão RC, Ventura LI. Estenose aórtica e doença coronariana. Análise dos fatores de risco. Arq Bras Cardiol 2006;87:115-20.

16. Vekshtein VI, Alexander RW, Yeung AC, Plappert T, St John Sutton MG, Ganz P et al. Coronary atherosclerosis is associated with left ventricular dysfunction and dilatation in aortic stenosis. Circulation 1990;82: 2068-74.

17. Bessone LN, Pupello DF, Hiro SP, LopezCuenca E, Glatterer MS, Ebra G. Surgical management of aortic valve disease in the elderly: a longitudinal analysis. Ann Thorac Surg 1988;.46:264-9. 
18. Vandeplas A, Willems JL, Piessens J, de Geest H. Frequency of angina pectoris and coronary artery disease in severe isolated valvular aortic stenosis. Am J Cardiol 1988;62: 117-20.

19. Sheiban J, Trevi GP, Benussi P, Marini A, Accardi R.; Di Bona E et al. Incidence of coronary artery disease in patients with valvular heart disease. Z Kardiol 1986;75(Suppl 2):76-9.

20. Ramsdale DR, Bennett DH, Bray CL, Ward C, Beton DC, Faragher EB. Angina, coronary risk factors and coronary artery disease in patients with valvular disease. A prospective study. Eur Heart J 1984;5:716-27.

21. Sokolow M, Lyon T P.

Eletrocardiographic diagnosis of left vntricular hypertrophy as obtained by unipolar, precordial and limbs leads. Am Heart J 1949; 37: 161-4.

22. Casale PN, Devereux RB, Alonso DR, Campo E, Kligfield P. Improved sexspecific criteria of left ventricular Hypetrophy for clinical and computer interpretation of electrocardiograms: validation with autopsy findings. Circulation 1987; 75: 565-72.

22. Devereux RB, Alonso DR, Lutas EM, et al. Echocardiografic assessment of left ventricular hypertrophy: comparison to necropsy findings. Am J Cardiol 1986; 57: 450-8.

23. Tribouilloy C, Shen WF, Peltier M, et al. Quantitation of aortic valve area in aortic stenosis with multiplane transesophageal echocardiography: comparison with monoplane transesophageal approach. Am Heart J 1994; 128: 526.

24. Aronow W S, Tresch D D, Nanna M. Aortic valve disease in the elderly. In: Tresch DD, Aronow WS, editors. Cardiovascular disease in the elderly patient. New York: Marcel Dekker, 1994: 345-72.

25. BraunwaldE. Valvular heart disease. In: Braunwald E., editors. A textbook of cardiovascular medicine. 7th ed. Philadelphia: WB Saunders; 2006. p. 1007-77.

26. Kelly DT. Assessment of aortic stenosis in the elderly. Cardiology in the Elderly 1995; 3: 159-60.

27. Roger VL, Tajib AJ, Bailey KR et al Progression of aortic stenosis em adults: new appraisal using Dopplerechocardiography. Am Heart J 1990; 119: 331-8.

28. Zak R. Factors contralling cardiac growth. In: Zak R. Growth of the heart and disease. New York: Ravin Press; 1984. p. 165-85.

29. Grossman W. Cardiac hypertrophy: useful adaptation or pathologic press? Am J Med 1980; 69: 576-84.

30. Carabello BA, Gren LH, Grossman W. Hemodynamic determinants of prognosis of aortic valve replacemente in critical aortic stenosis and advanced congestive heart failure. Circulation 1980; 62: 42-8.

31. Nunley DL, Grunkemerer GL, Starr A. Aortic valve replacement with coronary bypass grafting. J Thorac Cardiovasc Surg 1983;85:705-11.

32. Reber PAD, Safir DO, Guadalajara JF Estenosis aortica calcificada, semiologia $\mathrm{y}$ aspectos diagnosticos no invasivos en el anciano. Arch Inst Cardiol Mex 1986; 56: 41-7.

33. FlegJL. Eletrocardiographic findings in older persons without clinical heart disiase. In: Tresch DD, Aronow WS, editors. Cardiovascular disease in the elderly patients. New York: Marcel Dekker; 1994. p. 43-59. 
34. Tresch DD. Atypic presentations of cardiovascular disorders in the elderly. Geriatrics 1987; 42: 31-46.

35. Roberts WC. The senile calcification syndrome. Am J Cardiol 1986; 58: 372-3.

36. Aronow WS, Schwatz KS, Koenigsberg M. Correlation of serum lipids, calcium and phosphorus, diabetes mellitus, aortic valve stenosis and history of systemic hypertension with presence or absence of mitral anular calcium in persons older than 62 years in long-term health care facility. Am J Cardiol 1987; 59: 381-2.
37. Carroll JD, Carroll EP, Feldman T et al Sex associated differences in left ventricular function in aortic stenosis of the elderly. Circulation 1992; 86: 1099- 107.

38. Acar J, Vahanian A, Dicimetiere PH, Berdah J, Aouate PH, Sienczewski JA et al. Should coronary arteriography be performed in all patients who undergo catheterization for valvular heart disease? Z Kardiol 1986;75(Suppl 2):53-60. 
1 